Document downloaded from:

http://hdl.handle.net/10251/149088

This paper must be cited as:

Lloret, A.; Martinez Fuentes, A.; Agustí Fonfría, M.; Badenes, ML.; Rios, G. (2017). Chromatin-associated regulation of sorbitol synthesis in flower buds of peach. Plant Molecular Biology. 95(4-5):507-517. https://doi.org/10.1007/s11103-017-0669-6

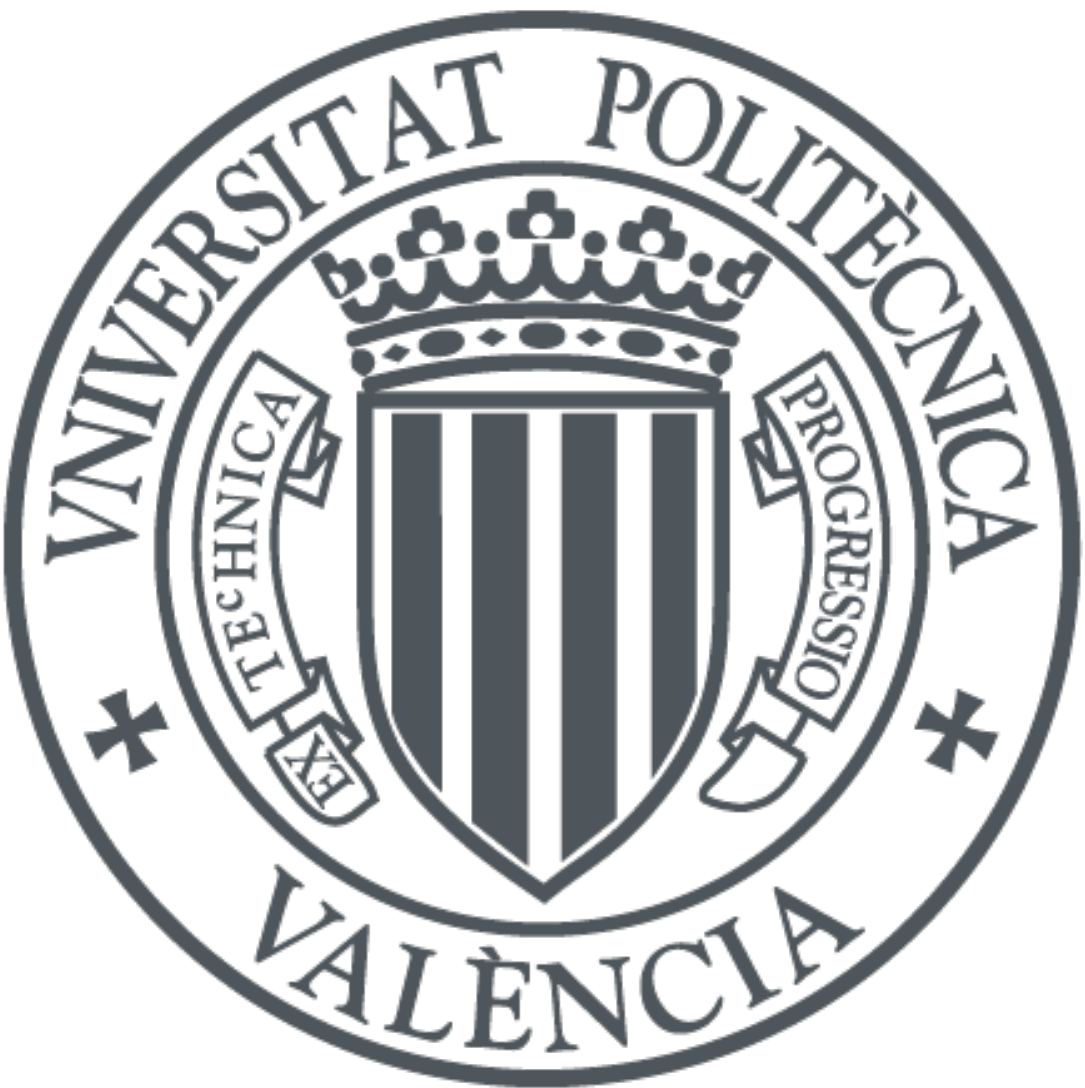

The final publication is available at

https://doi.org/10.1007/s11103-017-0669-6

Copyright Springer-Verlag

Additional Information 


\title{
Chromatin-associated regulation of sorbitol synthesis in flower
}

\section{buds of peach}

\author{
Alba Lloret ${ }^{1}$, Amparo Martínez-Fuentes ${ }^{2}$, Manuel Agustí ${ }^{2}$, María Luisa Badenes ${ }^{1}$, Gabino Ríos $^{1, *}$ \\ ${ }^{1}$ Instituto Valenciano de Investigaciones Agrarias (IVIA), Carretera CV-315, Km 10.7, E-46113 \\ Moncada, Valencia, Spain \\ ${ }^{2}$ Instituto Agroforestal Mediterráneo, Universitat Politécnica de Valéncia, Camino Vera s/n, E-46022 \\ Valencia, Spain \\ *Corresponding author \\ E-mail address: rios_gab@gva.es \\ Telephone number: +34963424000
}

\section{Key message}

PpeS6PDH gene is postulated to mediate sorbitol synthesis in flower buds of peach concomitantly with specific chromatin modifications.

\begin{abstract}
Perennial plants have evolved an adaptive mechanism involving protection of meristems within specialized structures named buds in order to survive low temperatures and water deprivation during winter. A seasonal period of dormancy further improves tolerance of buds to environmental stresses through specific mechanisms poorly known at the molecular level. We have shown that peach PpeS6PDH gene is down-regulated in flower buds after dormancy release, concomitantly with changes in the methylation level at specific lysine residues of histone H3 (H3K27 and H3K4) in the chromatin around the translation start site of the gene. PpeS6PDH encodes a NADPH-dependent sorbitol-6-phosphate dehydrogenase, the key enzyme for biosynthesis of sorbitol. Consistently, sorbitol accumulates in dormant buds showing higher PpeS6PDH expression. Moreover, PpeS6PDH gene expression is affected by cold and water deficit stress. Particularly, its expression is up-regulated by low temperature in buds and leaves, whereas desiccation treatment induces PpeS6PDH in buds and represses the gene in leaves. These data reveal the concurrent participation of chromatin modification mechanisms, transcriptional regulation of PpeS6PDH and sorbitol accumulation in flower buds of peach. In addition to its role as a major translocatable photosynthate in Rosaceae species, sorbitol is a widespread compatible solute and cryoprotectant, which suggests its participation in tolerance to environmental stresses in flower buds of peach.
\end{abstract}

\section{Keywords}

Abiotic stress, bud dormancy, chromatin, Prunus persica (peach), sorbitol-6-phosphate dehydrogenase (S6PDH), aldose-6-phosphate reductase (Ald6PRase) 


\section{Introduction}

Adaptation of perennial plants from temperate climates to seasonal fluctuations in temperature relies on bud set-up and dormancy (with the meaning of endodormancy throughout the text) among other adaptive improvements. Dormancy ensures the survival of vegetative and reproductive meristems in a quiescent state, which is released after the fulfilment of bud-intrinsic requirements of chilling, resembling the vernalization process described in Arabidopsis thaliana and cereals (Chouard 1960; Horvath et al. 2003). After achievement of chilling requirements and subsequent dormancy release, buds enter in an ecodormant stage requiring of a period of warm temperatures for proper bud-burst and flowering (Couvillon and Erez 1985). Buds undergoing dormancy are more tolerant to low and freezing temperatures and to desiccation, in virtue of physiological and molecular mechanisms that are insufficiently known, and that could partially overlap with those implicated in bud dormancy regulation (Fennell 2014; Wisniewski et al. 2015).

Among other, several epigenetic mechanisms involving genomic DNA methylation (Santamaría et al. 2009; Kumar et al. 2016a), histone modifications (Horvath et al. 2010; Leida et al. 2012; Ríos et al. 2014; Saito et al. 2015) and small RNAs production (Bai et al. 2016; Niu et al. 2016) have been postulated to mediate dormancy-dependent regulation of gene expression. Foremost targets of those regulatory pathways are DORMANCY-ASSOCIATED MADS-box (DAM) genes, identified as key transcriptional factors modulating bud dormancy in leafy spurge and stone-fruit tree species (Bielenberg et al. 2008; Horvath et al. 2010; Niu et al. 2016). Four from six tandemly arrayed DAM genes in peach are specifically modified by trimethylation of histone $\mathrm{H} 3$ in lysine 27 residue (H3K27me3) at specific genomic regions (de la Fuente et al. 2015). In spite of such burst of dormancy literature, supporting evidence on the participation of similar epigenetic pathways in the regulation of stress-related genes along bud development is still lacking.

Sorbitol is the primary photosynthetic product and the major phloem-translocated form of carbon in the Rosaceae (Webb and Burley 1962; Bieleski 1969). This sugar alcohol, or polyol, has been proposed to perform a protective role against stresses, acting as cryoprotectant, osmolyte and compatible solute under freezing, osmotic and water stress, respectively (Bieleski 1982; Loescher 1987; Escobar-Gutiérrez and Gaudillère 1996). Drought stress increases sorbitol accumulation in peach, although its participation in osmotic adjustment is a matter of controversy (Escobar-Gutiérrez et al. 1998; Lo Bianco et al. 2000). Sorbitol is produced in source tissues (photosynthetic leaves) via reduction of glucose-6-phosphate to sorbitol-6-phosphate by NADPH-dependent sorbitol-6-phosphate dehydrogenase (S6PDH), and the subsequent dephosphorylation of sorbitol-6-phosphate by a specific phosphatase, whereas sorbitol utilization occurs in sink tissues (Grant and ap Rees 1981; Loescher 1982). Since the first cloning of S6PDH gene from apple (Kanayama et al. 1992), many S6PDH have been identified based on sequence similarity to this gene, however few of them have been characterized at the enzymatic level. The overexpression of S6PDH from apple in transgenic tobacco increases sorbitol content and induces necrotic lesions in some cases (Tao et al. 1995; Sheveleva et al. 1998), whereas S6PDH silencing in apple reduces sorbitol accumulation and alters carbon partitioning (Teo et al. 2006). Moreover, the expression of $\mathrm{S} 6 \mathrm{PDH}$ is up-regulated by abscisic acid, low temperature and $\mathrm{NaCl}$ treatments in apple (Kanayama et 
al. 2006). Interestingly, expression of $S 6 P D H$ from apple in a Saccharomyces cerevisiae mutant deficient in synthesis of the osmolyte glycerol partially restores the tolerance to high $\mathrm{NaCl}$ concentrations (Shen et al. 1999). Also, overproduction of sorbitol by the expression of apple $S 6 P D H$ confers $\mathrm{NaCl}$ tolerance in transgenic Japanese persimmon (Gao et al. 2001).

Very recently, an ortholog of S6PDH has been cloned in peach, and its encoded protein (PpeAld6PRase) has been purified and extensively characterized at the enzymatic level (Hartman et al. 2017). In this work we refer to the gene encoding PpeAld6PRase as PpeS6PDH, following nomenclature suggestions of the Genome Database for Rosaceae (https://www.rosaceae.org/gene_class_listing). PpeS6PDH was unexpectedly expressed in dormant flower buds, a sink tissue, in a developmentally regulated manner. We have postulated its participation in the environmental and developmental dependent synthesis of sorbitol in buds.

\section{Materials and methods}

\section{Plant material and stress treatments}

Peach (Prunus persica [L.] Batsch) plants that were employed in this study were grown at Instituto Valenciano de Investigaciones Agrarias (IVIA) located in Moncada (Spain). Chilling requirements for dormancy release of the cultivars under study were previously estimated (Leida et al. 2012), and expressed in chilling hours $\left(\mathrm{CH}\right.$, hours at $\left.<7^{\circ} \mathrm{C}\right)$ required to have a bud break percentage higher than 50 $\%$ under forcing conditions. Interval estimates were as follows: 'Red Candem' $<278 \mathrm{CH}$, 'Crimson Baby' 412-511 CH, and 'Big Top' 674-712 CH. Collecting dates, cold accumulation and bud break percentage under forcing conditions of samples used for expression analysis and chromatin studies were respectively the following: 'Big Top' samples BT1 (November 3, 0 CH, 0 \%), BT2 (December 29, $276 \mathrm{CH}, 0$ \%), BT3 (January 12, 385 CH, 0 \%), BT4 (February 16, 634 CH, 27 \%), BT5 (March 2, 684 CH, 97 \%), and 'Red Candem' samples RC1 (November 3, 0 CH, 0 \%), RC2 (December 1, 50 CH, 0 \%), RC3 (December 15, $187 \mathrm{CH}, 0 \%$ ), and RC4 (December 29, $276 \mathrm{CH}, 89 \%$ ). As bud break percentages under forcing conditions of BT5 and RC4 were higher than $50 \%$, chilling requirements for dormancy release in these samples were considered fulfilled (Leida et al. 2012).

Samples required for tissue gene expression analysis (cv. 'Big Top') were obtained from flower buds (collected on January 12, 2010), leaves (November 6, 2012), embryos, flower parts (March 26, 2010) and fruit tissues (June 29, 2010).

For expression analysis in buds under stress conditions, dormant buds (November 3, 2015) and dormancy-released buds (January 25, 2016) of cv. 'Crimson Baby' were collected from three different trees. Six budsticks for each treatment were placed in glass tubes with $25 \mathrm{ml}$ of water at $25^{\circ} \mathrm{C}$ (control) during one and three days. Temperature stress incubations were made at $37{ }^{\circ} \mathrm{C}$ and $4{ }^{\circ} \mathrm{C}$, salinity stress was made by adding $200 \mathrm{mM} \mathrm{NaCl}$, and desiccation stress was performed without water. Routinely the base of budsticks was cut and the solution replaced with fresh one after two days incubation.

A stress analysis was carried out on leaf discs as described previously (Trotel et al. 1996). Ten discs of 1 cm of diameter per treatment were excised from five different trees of cv. 'Big Top' (June 9, 2015) and were incubated in $5 \mathrm{mM}$ HEPES, $1.5 \mathrm{mM} \mathrm{CaCl}_{2}$, and $10 \mathrm{mM} \mathrm{KCl}$ solution at $25^{\circ} \mathrm{C}$ (control). After $4 \mathrm{~h}$ incubation, discs were transferred to fresh solution with $250 \mathrm{mM} \mathrm{NaCl}$ for salt stress treatment, or 
incubated at $37{ }^{\circ} \mathrm{C}$ or $4{ }^{\circ} \mathrm{C}$ for temperature stress. Discs were collected at $4 \mathrm{~h}$ and $24 \mathrm{~h}$.

For the desiccation assay on leaves, adult leaves from three different trees of cv. 'Red Candem' (April 27, 2015 ) were placed into glasses with the petiole in contact with water (control) or without water (stressed samples), for one, three and seven days.

\section{Cloning of PpeS6PDH in pET-derived vectors}

The PpeS6PDH gene was cloned into the expression vectors pET302/NT-His and pET303/CT-His (Invitrogen), which facilitates the purification of the recombinant protein. For that, $P p e S 6 P D H$ was amplified using cDNA from peach flower buds collected on January 12 of 2010. The Phusion HighFidelity DNA Polymerase (Thermo Scientific) was used under the following PCR conditions: $2 \mathrm{~min}$ at $94{ }^{\circ} \mathrm{C}, 5$ cycles of $30 \mathrm{~s}$ at $94{ }^{\circ} \mathrm{C}, 30 \mathrm{~s}$ at $58^{\circ} \mathrm{C}$, and $1 \mathrm{~min}$ at $72{ }^{\circ} \mathrm{C}$, followed by 30 cycles of $30 \mathrm{~s}$ at $94{ }^{\circ} \mathrm{C}$, $30 \mathrm{~s}$ at $65^{\circ} \mathrm{C}$ and $1 \mathrm{~min}$ at $72{ }^{\circ} \mathrm{C}$, and a final step of $5 \mathrm{~min}$ at $72{ }^{\circ} \mathrm{C}$. All the primers used in this study are listed in Online Resource Table S1. The PCR product was purified with High Pure PCR Product Purification Kit (Roche) and digested with enzymes XhoI and BamHI (Roche) to have an N-terminal His tag or with $\mathrm{XhoI}$ and $\mathrm{Xba \textrm {I }}$ (Roche) for a C-terminal His tag. The purified product and corresponding vectors were ligated with T4 DNA ligase (Roche) and cloned into Escherichia coli BL21 (DE3) (Novagen). The nucleotide sequence of the inserted gene was confirmed by sequencing.

\section{Phylogenetic analysis of PpeS6PDH protein}

For the phylogenetic analysis, PpeS6PDH, S6PDH of Malus domestica (Kanayama et al. 1992), Pyrus Pyrifolia (Liu et al. 2013), Oryza sativa (Yadav and Prasad 2014) and other S6PDH-like proteins were used. In addition, mannose-6-phosphate reductase (M6PR) of Apium graveolens (Everard et al. 1997) and M6PR-like proteins were also included. M6PRs and S6PDHs belong to the same superfamily of aldo-keto reductases (Hyndman et al. 2003; Yadav and Prasad 2014) and keep high similarity (Bortiri et al. 2002). Sequence alignments were performed by ClustalW (Larkin et al. 2007), and Gblocks (Talavera and Castresana 2007) was used to remove wrong aligned regions. To build a phylogenetic tree, MEGA7 (Kumar et al. 2016b) was used with Maximum Likelihood method and tested using a Bootstrap with 1000 replicates.

\section{Expression and purification of recombinant protein}

In order to express the His-tagged PpeS6PDH, 50mL of LB medium supplemented with antibiotic was inoculated with 1/20 of an overnight culture of transformed E. coli BL21 (DE3) cells and incubated at 37 ${ }^{\circ} \mathrm{C}$ in an orbital shaker until the $\mathrm{OD}_{600} \sim 0.6$. Then the expression of recombinant protein was induced by adding $1 \mathrm{mM}$ isopropyl- $\beta$-D-thiogalactoside (IPTG; Roche) and incubated for $2 \mathrm{~h}$ at $37^{\circ} \mathrm{C}$. Induced BL21 (DE3) cells were harvested by centrifugation at $10,000 \mathrm{x}$ g during $10 \mathrm{~min}$ at $4{ }^{\circ} \mathrm{C}$. The enzyme was extracted using Bugbuster Plus Lysonase Kit (Novagen) and purified with PureProteome Nickel Magnetic Beads (Novagen), according to manufacture's instructions in both cases. Protein concentration was then measured with the Protein Quantification Kit-Rapid (Fluka) using bovine serum albumin as a standard. Protein size was determined by sodium dodecyl sulfate-polyacrylamide gel electrophoresis (SDS-PAGE) on $15 \%$ resolving gel and $3.5 \%$ stacking gel (Laemmli 1970). Protein bands were stained using 
Coomassie brilliant blue R-250.

\section{Enzymatic activity assay}

The enzymatic activity of PpeS6PDH was calculated as described previously (Yadav and Prasad 2014) with minor modifications. The assay solution contained $100 \mathrm{mM}$ Tris- $\mathrm{HCl}(\mathrm{pH} 8), 0.2 \mathrm{mM}$ NADPH, 50 $\mathrm{mM}$ of substrate (glucose-6-phosphate or mannose-6-phosphate), and $2.5 \mu \mathrm{g} / \mathrm{mL}$ of recombinant protein in a final volume of $0.8 \mathrm{~mL}$. The decrease of absorbance at $340 \mathrm{~nm}$ was measured. The assay was repeated three times for each condition.

\section{Expression analysis by real-time quantitative PCR}

For PpeS6PDH gene expression analysis, total RNA was isolated using the RNeasy Plant Mini Kit (Qiagen) and purified with the RNase-Free DNase Set (Qiagen), according to manufacturer's instructions with minor modifications. Polyvinylpyrrolidone (PVP-40) $1 \%(\mathrm{w} / \mathrm{v})$ was added to the kit extraction buffer before use. Then, RNA (500 ng) was reverse transcribed with PrimeScript RT reagent kit (Takara Bio) and cDNA was analyzed by quantitative real-time PCR on a StepOnePlus Real-Time PCR System (Life Technologies), utilizing SYBR premix Ex Taq (Tli RNaseH plus) (Takara Bio). Cycling conditions were $10 \mathrm{~min}$ at $95^{\circ} \mathrm{C}$, followed by 40 cycles of $15 \mathrm{~s}$ at $95^{\circ} \mathrm{C}$, and $1 \mathrm{~min}$ at $60^{\circ} \mathrm{C}$. Specificity of the amplification was evaluated by the presence of a single peak in the dissociation curve after PCR and by size estimation of the amplified product in agarose gel. Relative expression was measured using a relative standard curve. Bestkeeper (Pfaffl et al. 2004), NormFinder (Andersen et al. 2004) and $\Delta \mathrm{Ct}$ (Silver et al. 2006) methods were used in a previous study in order to determine the most stable housekeeping genes (Lloret et al. 2017). According to this study, actin-like and tubulin-like genes were used as references for tissue-dependent expression, actin-like and SAND-like for bud development samples, and SAND-like for stress treatments (Online Resource Table S1). When two reference genes were required for the analysis, the normalization factor was calculated by the geometric mean of the values of both genes. Results were the average of three independent biological replicates.

\section{Chromatin immunoprecipitation (ChIP) assays}

The chromatin immunoprecipitation method and 'Big Top' samples collected along bud development have been described previously (Leida et al. 2012). Those preceding immunoprecipitated samples were employed in real-time quantitative PCR assays using primers listed in Online Resource Table S1 and following PCR conditions shown above. The enrichment in H3K27me3 and H3K4me3 modifications present in PpeS6PDH gene were made relative to $\mathrm{H} 3$ values. Results were the average of three independent biological replicates with three technical replicates.

\section{Measurement of sugars and sorbitol content in buds}

Soluble sugars and sorbitol were analyzed as previously described (Eshghi et al. 2007) with minor modifications. Ground and dried buds of cv 'Red Candem' (100 mg) were mixed with $5 \mathrm{~mL}$ of petrolium ether $\left(40-60^{\circ}\right)$ and centrifuged at $1,109 \mathrm{x}$ for $10 \mathrm{~min}$ at $4{ }^{\circ} \mathrm{C}$. Then, petrolium ether containing lipids, chlorophyll and other contaminants was removed and $4 \mathrm{~mL}$ of ethanol $80 \%$ and $0.1 \mathrm{~mL}$ of mannitol (60 
$\mathrm{mg} / \mathrm{mL}$ ) were added to the pellet. Mannitol was used as an internal control. The samples were incubated during $20 \mathrm{~min}$ at $65^{\circ} \mathrm{C}$. After centrifugation for $5 \mathrm{~min}$ at $1,109 \mathrm{xg}$ at $4{ }^{\circ} \mathrm{C}$, the supernatant was recovered and the remaining plant material was then re-extracted with ethanol twice. All supernatants were mixed and dried again. For dried residue purification, $4 \mathrm{~mL}$ of $\mathrm{H}_{2} \mathrm{O}$ MiliQ and $20 \mu \mathrm{g}$ of activated charcoal were added and then, after another centrifugation, the supernatant was recovered and filtered through a nylon membrane $(0.45 \mu \mathrm{m})$. Finally, the samples were diluted and injected in a HPLC (High Performance Liquid Cromatography Spectra System) with CarboSep COREGEL-87 (Transgenomic) and a refractive index detector. The mobile phase (ultrapure water) was supplied at a flow rate of $0.6 \mathrm{~mL} / \mathrm{min}$. Standard solutions containing glucose, fructose, sorbitol, sucrose and mannitol at different concentrations were injected into the column and their peaks were used to construct calibration curves for each compound. The concentration of individual sugars and sugar alcohols in each tissue sample was then calculated using peak areas and the calibration curves. Results were the average of two independent replicates assayed twice.

\section{Statistical analysis}

Statistical analyses were performed using the Statgraphics XVI.I package 324 (Statpoint Technologies). The means of two samples were compared using non-parametric Man-Whitney $U$ test and comparisons of multiple samples were evaluated by non-parametric Klustal-Wallis test with a confidence level of $95 \%$. Significant differences between samples were labelled with asterisks or different letters.

\section{Results}

\section{Characterization of S6PDH protein in peach}

In previous transcriptomic studies we have identified some expressed sequence tags (GenBank accessions GR410685 and JK006377) corresponding to a S6PDH-like gene with differential expression along the development of flower buds of peach (Leida et al. 2010, 2012). The International Peach Genome Initiative (Verde et al. 2013) named this gene ppa009007m (v1.0) and Prupe.8G083400 (v2.1). In this study we will refer to ppa009007m gene as PpeS6PDH, according to the standard gene nomenclature in the Rosaceae (Jung et al. 2015) and suggestions of the Genome Database for Rosaceae (https://www.rosaceae.org/gene_class_listing).

The PCR-amplified coding DNA sequence of PpeS6PDH was identical to ppa009007m and to the mRNA coding for an Ald6PRase enzyme recently characterized (Hartman et al. 2017). The 310 amino acids long PpeS6PDH protein shared $76 \%$ identity with NADPH-dependent sorbitol-6-phosphate dehydrogenase from Malus domestica and 62\% identity with NADPH-dependent sorbitol-6-phosphate dehydrogenase from Oryza sativa, both enzymes well characterized. The phylogenetic analysis showed that PpeS6PDH is very close to S6PDH-like proteins from the Prunus genus and to other well characterized S6PDH proteins from the Rosaceae family (Malus domestica and Pyrus pyrifolia) (Fig. 1; Hartman et al. 2017). The gene has been recently cloned with an N-terminal His-tag fusion into pET19b vector, and the recombinant protein purified and characterized at the enzymatic level, showing a NADPH-dependent reductase activity on glucose-6-phosphate to produce sorbitol-6-phosphate (Hartman et al. 2017). We independently amplified PpeS6PDH gene and cloned it into pET302/NT-His vector inserting a 6xHis tag 
at the N-terminal end of the protein and pET303/CT-His leading to a C-terminal 6xHis tag. The SDSPAGE analysis of the purified recombinant protein showed a band between $29 \mathrm{kDa}$ and $47 \mathrm{kDa}$ markers, which was in concordance with the expected molecular mass of $36.7 \mathrm{kDa}$ (Online Resource Fig. S1). In a specific enzymatic assay, purified His-PpeS6PDH reduced glucose-6-phosphate to sorbitol-6-phosphate using NADPH as electron donor with a specific activity of $2.95 \mathrm{U} / \mathrm{mg}$ (Table 1), slightly higher than the activity described for related S6PDHs fused to N-terminal His tags from rice and apple (Figueroa and Iglesias 2010; Yadav and Prasad 2014), and very similar to the Vmax parameter found by Hartman et al. (2017) in their particular His-PpeS6PDH preparations. Reduction of mannose-6-phosphate occurred at a much lower specific activity of $0.07 \mathrm{U} / \mathrm{mg}$. We could not detect the enzymatic activity of PpeS6PDH with a His tag at the C-terminus, as similarly observed in apple S6PDH (Figueroa and Iglesias 2010). Taken together, these results confirmed that PpeS6PDH is able to perform sorbitol-6-phosphate dehydrogenase activity in vitro and that short fusions at the $\mathrm{C}$ terminus abolish PpeS6PDH activity.

\section{PpeS6PDH expression is developmentally regulated in buds}

To properly characterize PpeS6PDH at the molecular level, its relative expression was studied in different peach tissues. PpeS6PDH showed higher expression in leaves, flower buds, petals and sepals (Fig. 2).

PpeS6PDH expression was also appreciable in other flower organs (stamens and carpels), but insignificant in fruit (skin and flesh) and embryo samples.

We estimated the expression profile of PpeS6PDH along bud development in two cultivars with different chilling requirements for bud dormancy. Results confirmed the down-regulation of PpeS6PDH in flower buds after dormancy release (Fig. 3d), as revealed by previous transcriptomic studies (Leida et al. 2010, 2012). Interestingly, PpeS6PDH expression peaked at different times in dormant samples of 'Red Candem' and 'Big Top' cultivars, in concordance with their different chilling requirements. The early cultivar 'Red Candem' showed maximal expression on December 1 (RC2 sample), while the medium cultivar 'Big Top' reached maximal levels of expression on January 12 (BT3 sample). The expression decreased drastically in dormancy released buds, on December 29 (RC4) and March 2 (BT5) in 'Red Candem' and 'Big Top' cultivars, respectively (Fig. 3d).

Interestingly, the 'Big Top' sample collected on December 29 (BT2) increased PpeS6PDH expression with respect to the sample collected on November 3 (BT1), contrarily to the strong reduction observed in 'Red Candem' in the same period of time. These data argue for a dormancy-dependent regulation of PpeS6PDH expression in flower buds, acting independently of putative environmental effects.

\section{Changes in PpeS6PDH expression associate with chromatin modifications}

We performed ChIP analysis in order to identify chromatin modifications at PpeS6PDH during dormancy release. A previous genome-wide study of $\mathrm{H} 3 \mathrm{~K} 27 \mathrm{me} 3$ enrichment in buds was used to identify this specific modification in PpeS6PDH locus (de la Fuente et al. 2015). We found that H3K27me3 was significantly enriched on the translation start region of PpeS6PDH locus in dormancy released buds (Fig. 3a). H3K27me3 modification has been found associated with silencing of gene expression in peach and other plant and animal species, in close agreement with the repression of PpeS6PDH expression in dormancy released buds (Fig. 3d). H3K27 trimethylated region in PpeS6PDH contained a repetitive GA 
dinucleotide motif (marked with asterisks in Fig. 3a), which has been found associated with H3K27me3 stretches in peach and Arabidopsis (Deng et al. 2013; de la Fuente et al. 2015). In order to confirm these data, we amplified by quantitative real-time PCR a fragment contained into the H3K27 trimethylated region in $\mathrm{H} 3 \mathrm{~K} 27 \mathrm{me} 3$ - and $\mathrm{H} 3 \mathrm{~K} 4 \mathrm{me} 3$-immunoprecipitated samples along bud development. A concomitant increase in $\mathrm{H} 3 \mathrm{~K} 27 \mathrm{me} 3$ and decrease in $\mathrm{H} 3 \mathrm{~K} 4 \mathrm{me} 3$ enrichment was observed in BT5 sample containing buds after dormancy release (Fig. 3b,c). Differences in H3K27me3 were not statistically significant despite BT5 showed a higher value in the three biological replicates, due to the high variability found in this sample. H3K27me3 modification in the region around the ATG of PpeS6PDH was paralleled by a slight decrease in trimethylation of $\mathrm{H} 3 \mathrm{~K} 4$, a modification usually associated with transcriptional activation.

The gene model ppa008399m has been proposed to overlap PpeS6PDH in their common 3'-UTRs and the last exon of PpeS6PDH (Fig. 3a). We measured ppa008399m gene expression along development of flower buds of 'Red Candem' and 'Big Top' (Fig. 3e). The expression of ppa008399m increased slightly during bud development, but transcript accumulation did not correlate inversely with PpeS6PDH expression (Fig. 3d). Consequently we did not obtain evidences of co-regulated expression.

\section{Sorbitol content increases along bud development}

The content of some sugars (sucrose, glucose and fructose) and the sugar alcohol sorbitol was determined at different stages of bud development. Glucose and fructose contents were not altered in the assay. In contrast, sucrose and sorbitol amounts increased along bud development until the sample previous to dormancy release. After dormancy release their content remained stable or decreased slightly (Fig. 4). The increase in sorbitol level was concordant with changes in PpeS6PDH expression during the first stages of bud development (Fig. 3d). After that point, the transcriptional repression of PpeS6PDH explained the interrupted accumulation of sorbitol.

\section{PpeS6PDH shows cold-inducible expression}

The response of PpeS6PDH expression to abiotic stresses was assayed in buds and leaves, since certain previous studies propose a protective role of sorbitol and S6PDH against environmental stresses.

Firstly, flower buds were exposed to temperature and water stresses during one and three days treatments. PpeS6PDH expression was highly up-regulated after desiccation and cold $\left(4{ }^{\circ} \mathrm{C}\right)$ stresses in both dormant and non-dormant buds (Fig. 5a,b). In addition, transcript accumulation slightly increased with the duration of the treatment in both cases. On the other hand, PpeS6PDH expression showed a complex behaviour under heat treatment $\left(37^{\circ} \mathrm{C}\right)$, being down-regulated in dormant buds and up-regulated in dormancy released buds (Fig. 5b). Finally, we could notice a decreased PpeS6PDH expression following saline stress in dormant buds (Fig. 5a).

In leaf discs, cold exposure induced PpeS6PDH expression at a level similar to buds (Fig. 5d). However, in excised leaves desiccation caused a drastic down-regulation of PpeS6PDH (Fig. 5e), in contrast to the opposite behaviour observed in buds. Neither saline nor heat treatments affected significantly PpeS6PDH expression in leaves (Fig. 5c,d). 


\section{Discussion}

A recent study by Hartman et al. (2017) has shown by phylogenetic and enzymatic analysis that peach PpeS6PDH encodes a NADPH-dependent aldose-6-phosphate reductase with specificity for the reduction of glucose-6-phosphate to sorbitol-6-phosphate (also referred as S6PDH). The enzyme was inhibited by several hexose-phosphates, orthophosphate and oxidizing agents, offering alternative pathways for enzyme regulation. In our study, we have obtained similar activity values of the His-PpeS6PDH recombinant protein, and have confirmed the inhibitory effect of C-terminal His fusions on S6PDH activity, as also observed in apple S6PDH (Figueroa and Iglesias 2010).

In many Rosaceae, S6PDH enzymes are involved in sorbitol synthesis in source tissues (photosynthetic leaves). Subsequently, sorbitol is translocated to sink tissues and converted to fructose by sorbitol dehydrogenase (SDH) enzymes (Loescher 1987). Thus S6PDH genes are expected to be mainly expressed in fully developed leaves, where photosynthesis takes place. However we have found that PpeS6PDH is highly expressed in dormant flower buds, a sink tissue, in line with sorbitol accumulation data (Fig. 3d; Fig. 4). These results suggest that the active biosynthesis of sorbitol in flower buds is mediated by the transcriptional activity of PpeS6PDH gene, however biochemical studies showing a concomitant accumulation of PpeS6PDH protein are not currently available. In pear, as a response to artificial chilling exposure, sorbitol, sucrose and hexoses accumulated in flower and vegetative buds concomitantly with starch hydrolysis, suggesting the utilization of starch reserves to synthesize soluble sugars and sorbitol during bud dormancy (Hussain et al. 2015). In another study, sucrose and stachyose/raffinose carbohydrates accumulated in vegetative buds of peach instead of sorbitol, but no data about flower buds were presented (Marquat et al. 1999). A high increase in sorbitol content was observed in xylem sap of Japanese pear in late December, around bud dormancy release date, which prompted the authors to postulate a role of soluble sugars and sorbitol in flower bud dormancy regulation (Ito et al. 2012). An independent increase of sorbitol and carbohydrates occurred in xylem sap under $0{ }^{\circ} \mathrm{C}$ treatment, suggesting a role of sugar accumulation in acquisition of freezing tolerance (Ito et al. 2013). Similarly, sorbitol and sucrose accumulation observed in this study could perform a protective role against water deficit and low temperature stresses in flower buds. In that case, PpeS6PDH could exert a key regulatory role in seasonal tolerance of buds to abiotic stresses through sorbitol production. Gene expression analyses provide further insight into PpeS6PDH function. PpeS6PDH is up-regulated in dormant buds and subsequently repressed in dormancy-released samples, while a fragment including the translation start codon and a (GA)n repeated stretch undergoes H3K27me3 chromatin modification (Fig. 3a). This modification, associated with gene expression silencing at specific loci, has been also proposed to mediate stable silencing of several $D A M$ genes, leading to bud dormancy release after the accomplishment of chilling requirements (Leida et al. 2012; de la Fuente et al. 2015). Interestingly, (GA)n repeat elements have been found enriched in $\mathrm{H} 3 \mathrm{~K} 27$ me 3 modified and FIE-binding regions in Arabidopsis (Deng et al. 2013), suggesting its participation in chromatin regulatory circuits. In addition to $\mathrm{H} 3 \mathrm{~K} 27 \mathrm{me} 3$ modification, PpeS6PDH chromatin associates with a decreased trimethylation of $\mathrm{H} 3 \mathrm{~K} 4$ along bud development and dormancy release, as similarly described at DAM6 locus (Leida et al. 2012). Both, H3K27me3 and H3K4me3 changes could account for specific and sequential steps of gene expression down-regulation, as formerly postulated in DAM6 (Ríos et al. 2014). Moreover, 
additional epigenetic modifications such as DNA methylation and others are expected to act coordinately with the observed histone changes. In fact, the occurrence of specific DNA methylation events has been reported in other tree species during bud dormancy and development (Kumar et al. 2016a, Conde et al. 2017).

Expression analysis of the gene adjacent to PpeS6PDH (ppa008399m) served to conclude that H3K27me3-associated silencing affected locally to PpeS6PDH instead of being sprayed to near genes, as was previously proposed in a genome-wide analysis studying H3K27me3 enrichment in buds (de la Fuente et al. 2015).

Up-regulation of PpeS6PDH expression by low temperature treatments in buds and leaves (Fig. 5b,d) confirmed its participation in the chilling response. Induction of $S 6 P D H$ expression by low temperature has been also observed in apple leaves (Kanayama et al. 2006; Liang et al. 2012). Interestingly, high temperature and desiccation treatments produced antagonistic effects in different samples. Particularly, desiccation induced PpeS6PDH expression in buds, whereas PpeS6PDH was strongly down-regulated in leaves (Fig. 5a,e). These differences reveal tissue-specific mechanisms of regulation that could respond to distinct source/sink roles. Different degrees of drought stress also caused a reduction in S6PDH enzymatic activity in leaves of peach, whereas SDH enzymatic activity in shoot tips (a sink tissue) decreased (Lo Bianco et al. 2000).

In our opinion, developmental and environmental issues affecting PpeS6PDH expression, in addition to sorbitol accumulation data, suggests a role of this gene in protection against abiotic stresses, particularly chilling and desiccation, in flower buds of peach. Moreover, down-regulation of PpeS6PDH in dormancy-released buds associates with chromatin modification mechanisms similarly to DAM6 gene, suggesting the participation of common regulatory factors in PpeS6PDH and bud dormancy regulation.

\section{Acknowledgements}

This work was funded by the Instituto Nacional de Investigación y Tecnología Agraria y Alimentaria (INIA)-FEDER (RF2013-00043-C02-02) and the Ministry of Science and Innovation of Spain (AGL2010-20595). AL was funded by a fellowship co-financed by the European Social Fund and the Instituto Valenciano de Investigaciones Agrarias (IVIA). We thank Matilde González, José Martínez and José Palanca for their technical assistance.

\section{Author contributions}

MLB and GR conceived the study. AL, AMF and GR performed the experimental work. MA provided supervision and designed the method for sugars measurements. AL and GR prepared the manuscript.

\section{Conflict of Interest}

The authors declare that they have no conflict of interest.

\section{References}

Andersen CL, Jensen JL, Ørntoft TF (2004) Normalization of real-time quantitative reverse transcriptionPCR data: a model-based variance estimation approach to identify genes suited for normalization, 
applied to bladder and colon cancer data sets. Cancer Res 64:5245-5250. doi: 10.1158/00085472.CAN-04-0496

Bai S, Saito T, Ito A et al (2016) Small RNA and PARE sequencing in flower bud reveal the involvement of sRNAs in endodormancy release of Japanese pear (Pyrus pyrifolia 'Kosui'). BMC Genomics 17:230. doi: 10.1186/s12864-016-2514-8

Bielenberg DG, Wang Y, Li Z et al (2008) Sequencing and annotation of the evergrowing locus in peach (Prunus persica [L.] Batsch) reveals a cluster of six MADS-box transcription factors as candidate genes for regulation of terminal bud formation. Tree Genet Genomes 4:495-507. doi: 10.1007/s1 1295-007-0126-9

Bieleski RL (1969) Accumulation and translocation of sorbitol in apple phloem. Aust J Biol Sci 22:611620. doi: 10.1071/BI9690611

Bieleski RL (1982) Sugar alcohols. In: Loewus F, Tanner W (ed) Encyclopedia of plant physiology, new series 13A, Springer-Verlag, Berlin, pp 158-192

Bortiri E, Oh SH, Gao FY, Potter D (2002) The phylogenetic utility of nucleotide sequences of sorbitol 6phosphate dehydrogenase in Prunus (Rosaceae). Am J Bot 89:1697-1708. doi: 10.3732/ajb.89.10.1697

Chouard P (1960) Vernalization and its relations to dormancy. Annu Rev Plant Physiol 11:191-238. doi: 10.1146/annurev.pp.11.060160.001203

Conde D, Le Gac AL, Perales M et al (2017) Chilling-responsive DEMETER-LIKE DNA demethylase mediates in poplar bud break. Plant Cell Environ 40:2236-2249. doi: 10.1111/pce.13019

Couvillon GA, Erez A (1985) Influence of prolonged exposure to chilling temperatures on bud break and heat requirement for bloom of several fruit species. J Amer Soc Hort Sci 110:47-50

de la Fuente L, Conesa A, Lloret A, Badenes ML, Ríos G (2015) Genome-wide changes in histone H3 lysine 27 trimethylation associated with bud dormancy release in peach. Tree Genet Genomes 11:45. doi: 10.1007/s11295-015-0869-7

Deng W, Buzas DM, Ying H et al (2013) Arabidopsis polycomb repressive complex 2 binding sites contain putative GAGA factor binding motifs within coding regions of genes. BMC Genomics 14:593. doi: 10.1186/1471-2164-14-593

Escobar-Gutiérrez AJ, Gaudillère JP (1996) Distribution, metabolism and role of sorbitol in higher plants. A review. Agronomie 16:281-298. doi: 10.1051/agro:19960502

Escobar-Gutiérrez AJ, Zipperlin B, Carbonne F, Moing A, Gaudillére JP (1998) Photosynthesis, carbon partitioning and metabolite content during drought stress in peach seedlings. Aust J Plant Physiol 25:197-205. doi: 10.1071/PP97121

Eshghi S, Tafazoli E, Dokhani S, Rahemi M, Emam Y (2007) Changes in carbohydrate contents in shoot tips, leaves and roots of strawberry (Fragaria $x$ ananassa Duch) during flower-bud differentiation. Sci Hortic 113:255-260. doi: 10.1016/j.scienta.2007.03.014

Everard JD, Cantini C, Grumet R, Plummer J, Loescher WH (1997) Molecular cloning of mannose-6phosphate reductase and its developmental expression in celery. Plant Physiol 113:1427-1435. doi: 10.1104/pp.113.4.1427

Fennell A (2014) Genomics and functional genomics of winter low temperature tolerance in temperate 
fruit crops. Crit Rev Plant Sci 33:125-140. doi: 10.1080/07352689.2014.870410

Figueroa CM, Iglesias AA (2010) Aldose-6-phosphate reductase from apple leaves: Importance of the quaternary structure for enzyme activity. Biochimie 92:81-88. doi: 10.1016/j.biochi.2009.09.013

Gao M, Tao R, Miura K, Dandekar AM, Sugiura A (2001) Transformation of Japanese persimmon (Diospyros kaki Thunb) with apple cDNA encoding NADP-dependent sorbitol-6-phosphate dehydrogenase. Plant Sci 160:837-845. doi: 10.1016/S0168-9452(00)00458-1

Grant CR, ap Rees T (1981) Sorbitol metabolism by apple seedlings. Phytochemistry 20:1505-1511. doi:10.1016/S0031-9422(00)98521-2

Hartman MD, Figueroa CM, Arias DG, Iglesias AA (2017) Inhibition of recombinant aldose-6-phosphate reductase from peach leaves by hexose-phosphates, inorganic phosphate and oxidants. Plant Cell Physiol 58:145-155. doi: 10.1093/pcp/pcw180

Horvath DP, Anderson JV, Chao WS, Foley ME (2003) Knowing when to grow: signals regulating bud dormancy. Trends Plant Sci 8:534-540. doi: 10.1016/j.tplants.2003.09.013

Horvath DP, Sung S, Kim D, Chao W, Anderson J (2010) Characterization, expression and function of DORMANCY ASSOCIATED MADS-BOX genes from leafy spurge. Plant Mol Biol 73:169-179. doi: $10.1007 / \mathrm{s} 11103-009-9596-5$

Hussain S, Niu Q, Yang F, Hussain N, Teng Y (2015) The possible role of chilling in floral and vegetative bud dormancy release in Pyrus pyrifolia. Biol Plant 59:726-734. doi: 10.1007/s10535015-0547-5

Hyndman D, Baumanb DR, Herediac VV, Penning TM (2003) The aldo-keto reductase superfamily homepage. Chem Biol Interact 143-144:621-631. doi: 10.1016/S0009-2797(02)00193-X

Ito A, Sakamoto D, Moriguchi T (2012) Carbohydrate metabolism and its possible roles in endodormancy transition in Japanese pear. Sci Hortic 144:187-194. doi: 10.1016/j.scienta.2012.07.009

Ito A, Sugiura T, Sakamoto D, Moriguchi T (2013) Effects of dormancy progression and low-temperature response on changes in the sorbitol concentration in xylem sap of Japanese pear during winter season. Tree Physiol 33:398-408. doi: 10.1093/treephys/tpt021

Jung S, Bassett C, Bielenberg DG et al (2015) A standard nomenclature for gene designation in the Rosaceae. Tree Genet Genomes 11:108. doi: 10.1007/s11295-015-0931-5

Kanayama Y, Mori H, Imaseki H, Yamaki S (1992) Nucleotide sequence of a cDNA encoding NADPsorbitol-6-phosphate dehydrogenase from apple. Plant Physiol 100:1607-1608.

Kanayama Y, Watanabe M, Moriguchi R, Deguchi M, Kanahama K, Yamaki S (2006) Effects of low temperature and abscisic acid on the expression of the sorbitol-6-phosphate dehydrogenase gene in apple leaves. J Japan Soc Hort Sci 75:20-25. doi: 10.2503/jjshs.75.20

Kumar G, Rattan UK, Singh AK (2016a) Chilling-mediated DNA methylation changes during dormancy and its release reveal the importance of epigenetic regulation during winter dormancy in apple (Malus $x$ domestica Borkh). PLoS One 11:e0149934. doi: 10.1371/journal.pone.0149934

Kumar S, Stecher G, Tamura K (2016b) MEGA7: Molecular Evolutionary Genetics Analysis version 70 for bigger datasets. Mol Biol Evol 33:1870-1874. doi: 10.1093/molbev/msw054

Laemmli UK (1970) Cleavage of structural proteins during the assembly of the head of bacteriophage T4. Nature 227:680-685. doi: 10.1038/227680a0 
Larkin MA, Blackshields G, Brown NP et al (2007) Clustal W and Clustal X version 2.0. Bioinformatics 23:2947-2948. doi: 10.1093/bioinformatics/btm404

Leida C, Conesa A, Llácer G, Badenes ML, Ríos G (2012) Histone modifications and expression of DAM6 gene in peach are modulated during bud dormancy release in a cultivar-dependent manner. New Phytol 193:67-80. doi: 10.1111/j.1469-8137.2011.03863.x

Leida C, Terol J, Martí G et al (2010) Identification of genes associated with bud dormancy release in Prunus persica by suppression subtractive hybridization. Tree Physiol 30:655-666. doi: 10.1093/treephys/tpq008

Liang D, Cui M, Wu S, Ma F-W (2012) Genomic structure, sub-cellular localization, and promoter analysis of the gene encoding sorbitol-6-phosphate dehydrogenase from apple. Plant Mol Biol Rep 30:904-914. doi: 10.1007/s11105-011-0409-z

Liu D, Ni J, Wu R, Teng Y (2013) High temperature alters sorbitol metabolism in Pyrus pyrifolia leaves and fruit flesh during late stages of fruit enlargement. J Am Soc Hortic Sci 138:443-451

Lloret A, Conejero A, Leida C et al (2017) Dual regulation of water retention and cell growth by a stressassociated protein (SAP) gene in Prunus. Sci Rep 7:332. doi: 10.1038/s41598-017-00471-7

Lo Bianco R, Rieger M, Sung S-JS (2000) Effect of drought on sorbitol and sucrose metabolism in sinks and sources of peach. Physiol Plant 108:71-78. doi: 10.1034/j.1399-3054.2000.108001071.x

Loescher WH (1987) Physiology and metabolism of sugar alcohols in higher-plants. Physiol Plant 70:553-557. doi: 10.1111/j.1399-3054.1987.tb02857.x

Loescher WH, Marlow GC, Kennedy RA (1982) Sorbitol metabolism and sink-source interconversions in developing apple leaves. Plant Physiol 70:335-339. doi: 10.1104/pp.70.2.335

Marquat C, Vandamme M, Gendraud M, Pétel G (1999) Dormancy in vegetative buds of peach: relation between carbohydrate absorption potentials and carbohydrate concentration in the bud during dormancy and its release. Sci Hortic 79:151-162. doi: 10.1016/S0304-4238(98)00203-9

Niu Q, Li J, Cai D et al (2016) Dormancy-associated MADS-box genes and microRNAs jointly control dormancy transition in pear (Pyrus pyrifolia white pear group) flower bud. J Exp Bot 67:239-257. doi: $10.1093 / \mathrm{jxb} / \mathrm{erv} 454$

Pfaffl MW, Tichopad A, Prgomet C, Neuvians TP (2004) Determination of stable housekeeping genes, differentially regulated target genes and sample integrity: BestKeeper - Excel-based tool using pair-wise correlations. Biotechnol Lett 26:509-515. doi: 10.1023/B:BILE.0000019559.84305.47

Ríos G, Leida C, Conejero A, Badenes ML (2014) Epigenetic regulation of bud dormancy events in perennial plants. Front Plant Sci 5:247. doi: 10.3389/fpls.2014.00247

Saito T, Bai S, Imai T, Ito A, Nakajima I, Moriguchi T (2015) Histone modification and signalling cascade of the dormancy-associated MADS-box gene, PpMADS13-1, in Japanese pear (Pyrus pyrifolia) during endodormancy. Plant Cell Environ 38:1157-1166. doi: 10.1111/pce.12469

Santamaría ME, Hasbún R, Valera MJ et al (2009) Acetylated H4 histone and genomic DNA methylation patterns during bud set and bud burst in Castanea sativa. J Plant Physiol 166:1360-1369. doi: 10.1016/j.jplph.2009.02.014

Shen B, Hohmann S, Jensen RG, Bohnert HJ (1999) Roles of sugar alcohols in osmotic stress adaptation Replacement of glycerol by mannitol and sorbitol in yeast. Plant Physiol 121:45-52. doi: 
10.1104/pp.121.1.45

Sheveleva EV, Marquez S, Chmara W, Zegeer A, Jensen RG, Bohnert HJ (1998) Sorbitol-6-phosphate dehydrogenase expression in transgenic tobacco High amounts of sorbitol lead to necrotic lesions. Plant Physiol 117:831-839. doi: 10.1104/pp.117.3.831

Silver N, Best S, Jian J, Thein SL (2006) Selection of housekeeping genes for gene expression studies in human reticulocytes using real-time PCR. BMC Mol Biol 7:33. doi: 10.1186/1471-2199-7-33

Talavera G, Castresana J (2007) Improvement of phylogenies after removing divergent and ambiguously aligned blocks from protein sequence alignments. Syst Biol 56:564-577. doi: $10.1080 / 10635150701472164$

Tao R, Uratsu SL, Dandekar AM (1995) Sorbitol synthesis in transgenic tobacco with apple cDNA encoding NADP-dependent sorbitol-6-phosphate dehydrogenase. Plant Cell Physiol 36:525-532. doi: 10.1093/oxfordjournals.pcp.a078789

Teo G, Suzuki Y, Uratsu SL et al (2006) Silencing leaf sorbitol synthesis alters long-distance partitioning and apple fruit quality. Proc Natl Acad Sci USA 103:18842-18847. doi: 10.1073/pnas.0605873103

Trotel P, Bouchereau A, Niogret MF, Larher F (1996) The fate of osmo-accumulated proline in leaf discs of Rape (Brassica napus L) incubated in a medium of low osmolarity. Plant Sci 118:31-45. doi: 10.1016/0168-9452(96)04422-6

Verde I, Abbott AG, Scalabrin S et al (2013) The high-quality draft genome of peach (Prunus persica) identifies unique patterns of genetic diversity, domestication and genome evolution. Nat Genet 45:487-494. doi: 10.1038/ng.2586

Webb KL, Burley JWA (1962) Sorbitol translocation in apple. Science 137:766 doi: 10.1126/science.137.3532.766

Wisniewski M, Norelli J, Artlip T (2015) Overexpression of a peach CBF gene in apple: a model for understanding the integration of growth, dormancy, and cold hardiness in woody plants. Front Plant Sci 6:85. doi: 10.3389/fpls.2015.00085

Yadav R, Prasad R (2014) Identification and functional characterization of sorbitol-6-phosphate dehydrogenase protein from rice and structural elucidation by in silico approach. Planta 240:223238. doi: 10.1007/s00425-014-2076-4 


\section{Figure legends}

Fig 1 Phylogenetic tree of S6PDH and related proteins. The tree was constructed using the Maximum Likelihood method and bootstrapped with 1000 replicates. The scale bar indicates the branch length that corresponds to the number of substitutions per amino acid position

Fig 2 Relative expression of PpeS6PDH by qRT-PCR. Samples included different plant tissues and organs obtained from 'Big Top' cultivar: leaf (Le), fruit skin, fruit flesh, sepal (Se), petal (Pe), stamen (St), carpel (Ca), embryo (Em) and dormant flower bud (FB, sample BT3). Tubulin-like and actin-like genes were used as reference genes. An expression value of one is assigned to the leaf sample. Data are means from three biological replicates, with error bars representing standard deviation. Different letters (a-e) indicate significant difference between samples with a confidence level of $95 \%$

Fig 3 Chromatin immunoprecipitation (ChIP) analysis of PpeS6PDH gene during bud development. (a) Local H3K27me3 enrichment in a genomic region including PpeS6PDH and the adjacent gene ppa008399m by ChIP-Seq analysis in dormant (BT1) and non-dormant (BT5) buds of 'Big Top', compared with input sample. A peak on the first exon of PpeS6PDH in the non-dormant sample indicates the presence of a differentially methylated region. Predicted PpeS6PDH and ppa008399m transcripts are shown (peach genome v1.0) with their respective coding sequences (white rectangles) and untranslated 5' and 3' regions (grey rectangles). Repeated (GA)n elements are labelled with asterisks. The fragment amplified by qRT-PCR with specific primers is labelled with a striped rectangle. H3K27me3 (b) and H3K4me3 (c) modifications around the translation start site in the first exon of PpeS6PDH have been confirmed by qRT-PCR of ChIP samples at different bud development stages ('Big Top' BT1-5, see below). $\mathrm{H} 3 \mathrm{~K} 27 \mathrm{me} 3$ and $\mathrm{H} 3 \mathrm{~K} 4 \mathrm{me} 3$ enrichment has been made relative to histone $\mathrm{H} 3$ immunoprecipitated samples. The relative expression level of PpeS6PDH (d) and ppa008399m (e) during bud development has been analyzed in cultivars 'Big Top' and 'Red Candem'. Bud samples from 'Big Top' and 'Red Candem' were collected in autumn and winter (2009/2010) at different dates: November 3 (BT1 and RC1), December 1 (RC2), December 15 (RC3), December 29 (BT2 and RC4), January 12 (BT3), February 16 (BT4) and March 2 (BT5). In BT5 and RC4 samples (dark bars in the graphs) dormancy was already released. SAND-like and actin-like genes were used as reference genes. An expression value of one is assigned to the first sample (BT1 or RC1). Data are means from three biological samples with three technical replicates each, with error bars representing standard deviation. Different letters (a-d) indicate significant difference between samples with a confidence level of $95 \%$

Fig 4 Sugar and sorbitol accumulation in buds. Sucrose, glucose, fructose and sorbitol were measured in flower bud samples RC1 (1), RC2 (2), RC3 (3) and RC4 (4) of 'Red Candem'. Sample code is explained in Figure 3. Dormancy has been released in RC4 (black bars). Different letters (a-c) indicate significant difference between samples with a confidence level of $95 \%$

Fig 5 Effect of abiotic stresses on PpeS6PDH relative expression by qRT-PCR. Dormant and non- 
dormant flower buds of peach (cv. 'Crimson Baby') were treated with $200 \mathrm{mM} \mathrm{NaCl}$ or desiccated (a), incubated at $4{ }^{\circ} \mathrm{C}$ or $37^{\circ} \mathrm{C}$ (b), for one (white bars) or three days (grey bars). Leaf discs from 'Big Top' cultivar were treated with $250 \mathrm{mM} \mathrm{NaCl}(\mathbf{c})$, incubated at $4{ }^{\circ} \mathrm{C}$ or $37^{\circ} \mathrm{C}(\mathbf{d})$, for $4 \mathrm{~h}$ (white bars) or $24 \mathrm{~h}$ (grey bars). In a last experiment (e), excised leaves from 'Red Candem' cultivar were desiccated for one (white bars), three (grey bars) or seven days (black bars). SAND-like gene was used as reference. An expression value of one is assigned to the untreated sample at the first time point, that is one day (panels a, b and e) and 4 hours (panels $\mathbf{c}$ and d). Data are means from three biological samples with two technical replicates each, with error bars representing standard deviation. An asterisk indicates significant difference with the untreated control at a confidence level of $95 \%$ 


\section{Tables}

Table 1. Enzymatic activity of recombinant PpeS6PDH

\begin{tabular}{ccccc}
\hline & Substrate & Activity $(\mathrm{mU})$ & $\begin{array}{c}\text { Protein } \\
(\mu \mathrm{g})\end{array}$ & $\begin{array}{c}\text { Specific activity } \\
(\mathrm{U} / \mathrm{mg})\end{array}$ \\
\hline His-PpeS6PDH & Glucose-6-phosphate & $8.85 \pm 0.81$ & 3.0 & $2.95 \pm 0.27$ \\
His-PpeS6PDH & Mannose-6-phosphate & $0.25 \pm 0.09$ & 3.0 & $0.07 \pm 0.03$ \\
PpeS6PDH-His & Glucose-6-phosphate & ND & 1.5 & - \\
\hline
\end{tabular}

ND, not detected

Fig. 1

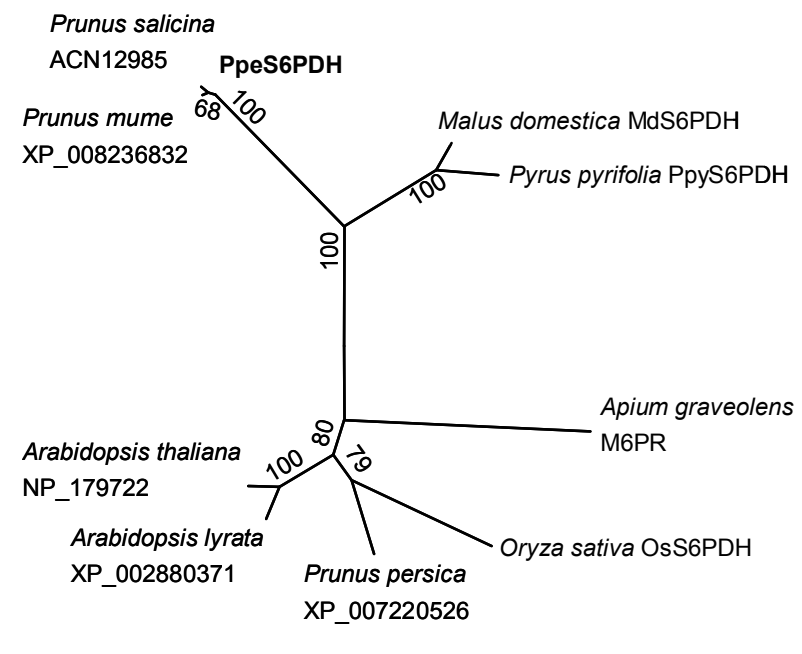

$\overrightarrow{0.05}$

Fig. 2

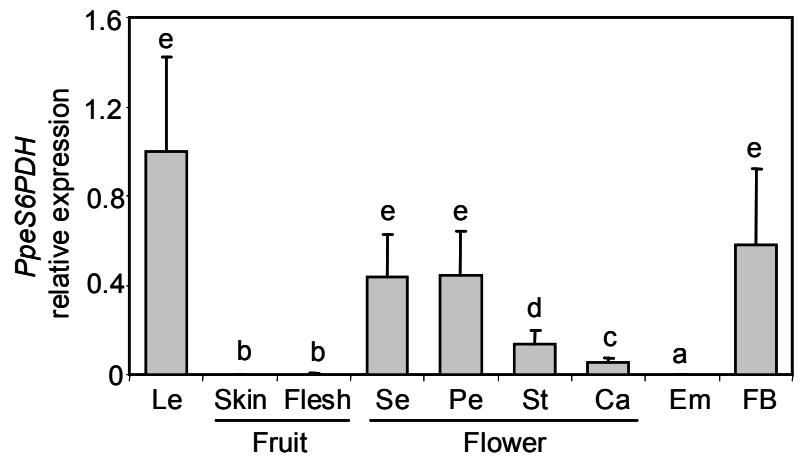


Fig. 3

(A)

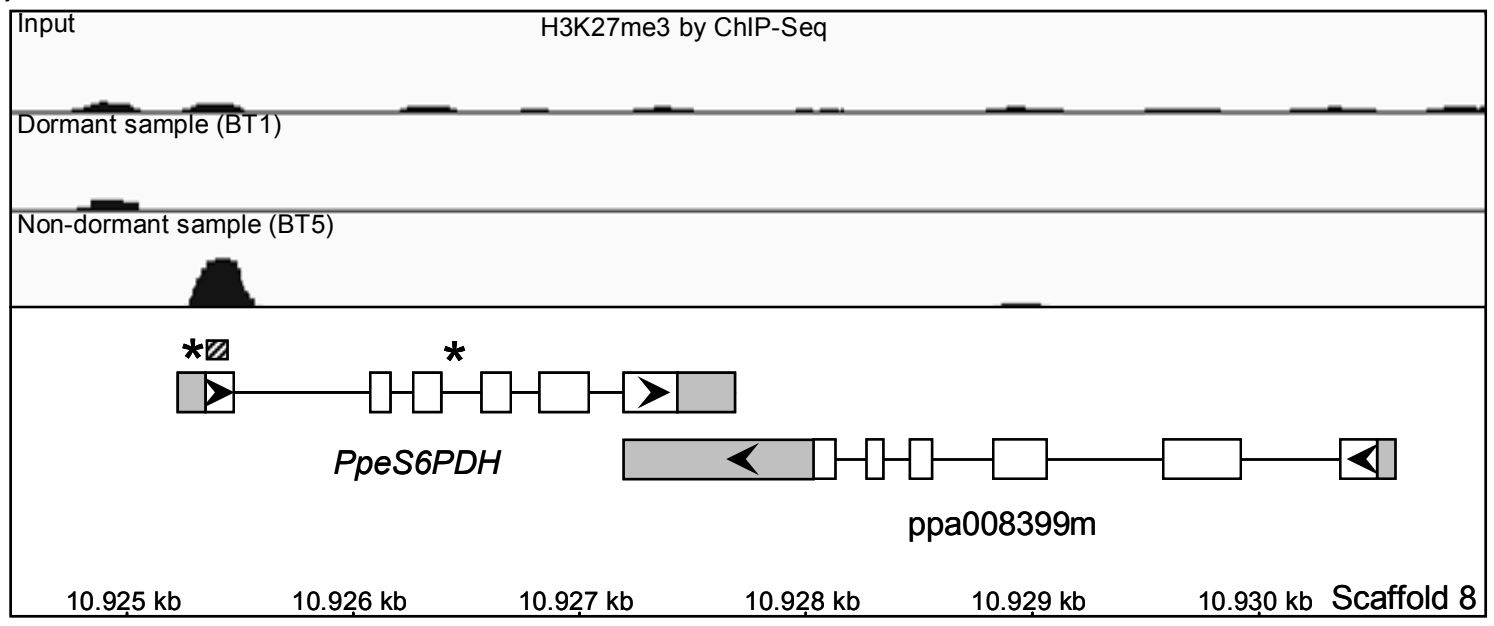

(B)

(C)
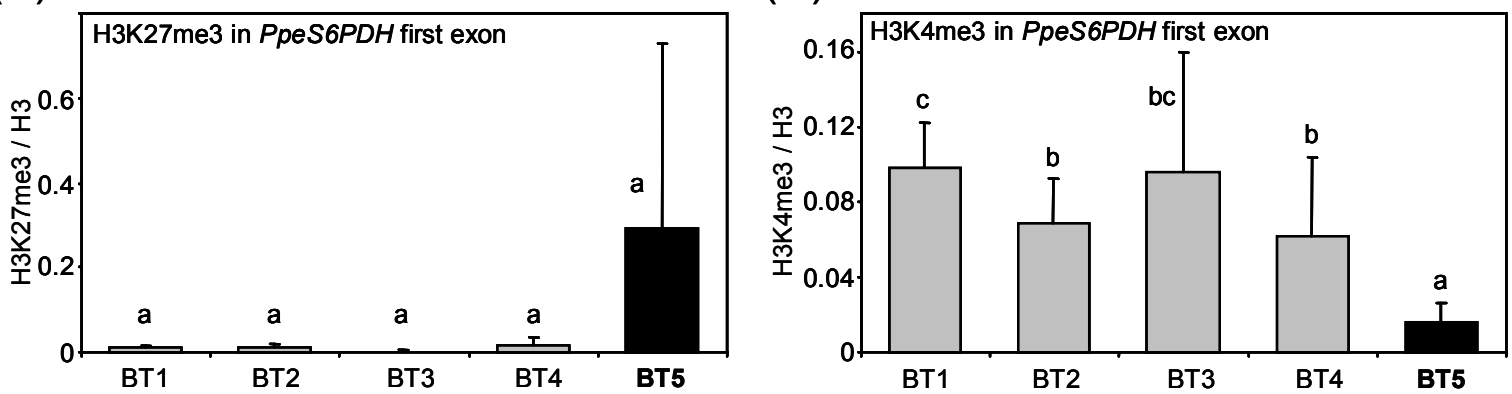

(D)

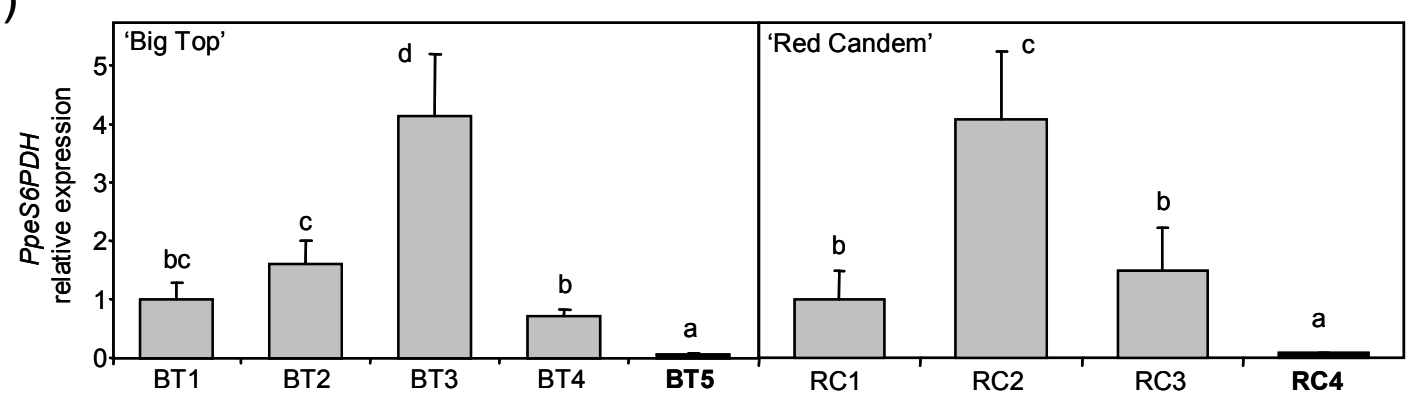

(E)

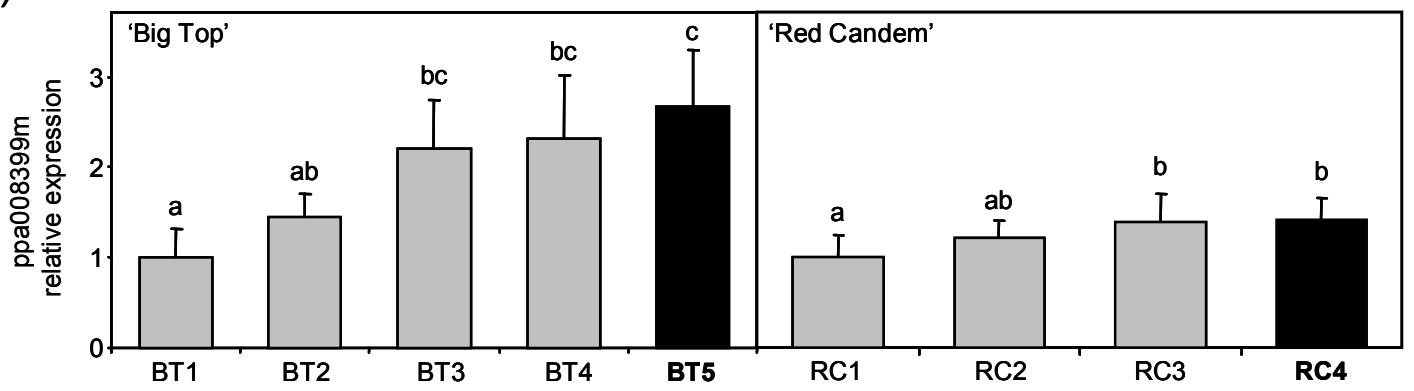


Fig. 4

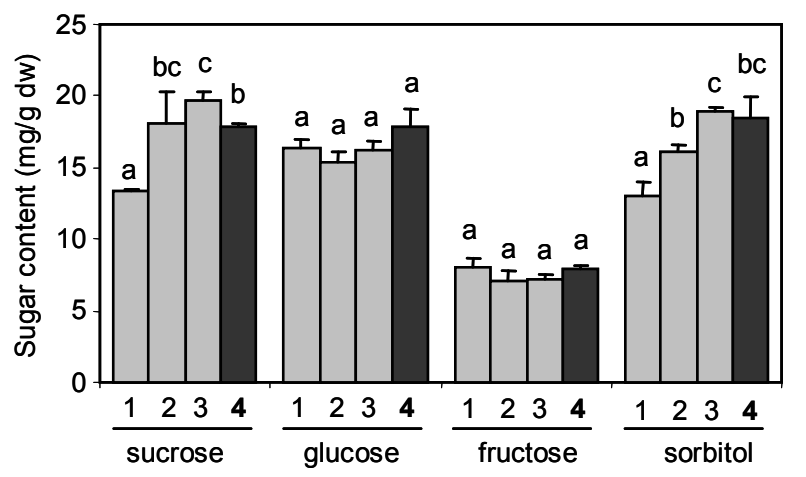

Fig. 5

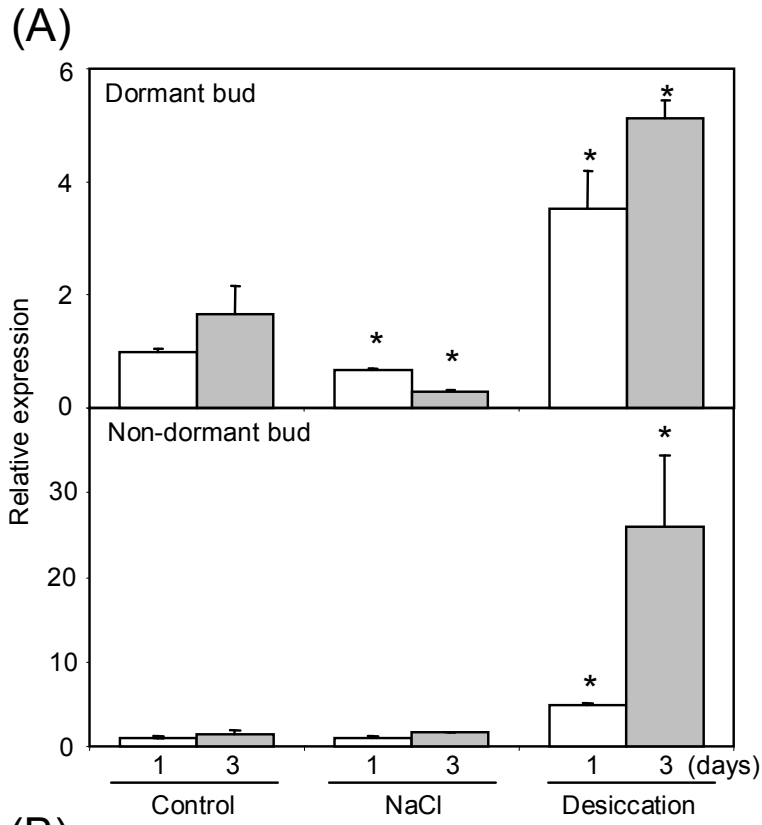

(C)

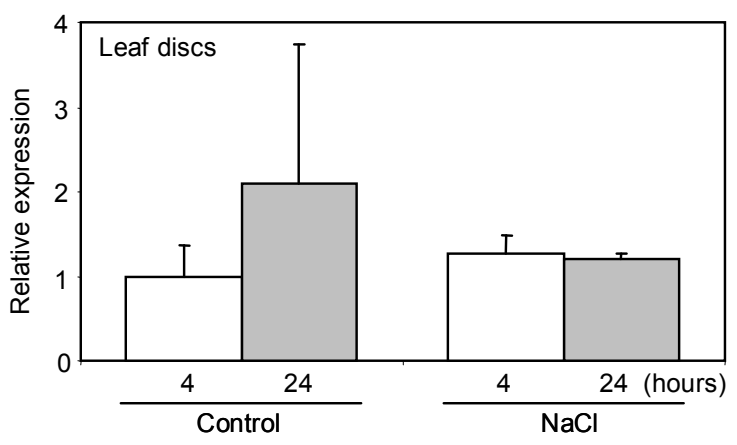

(D)

(B)
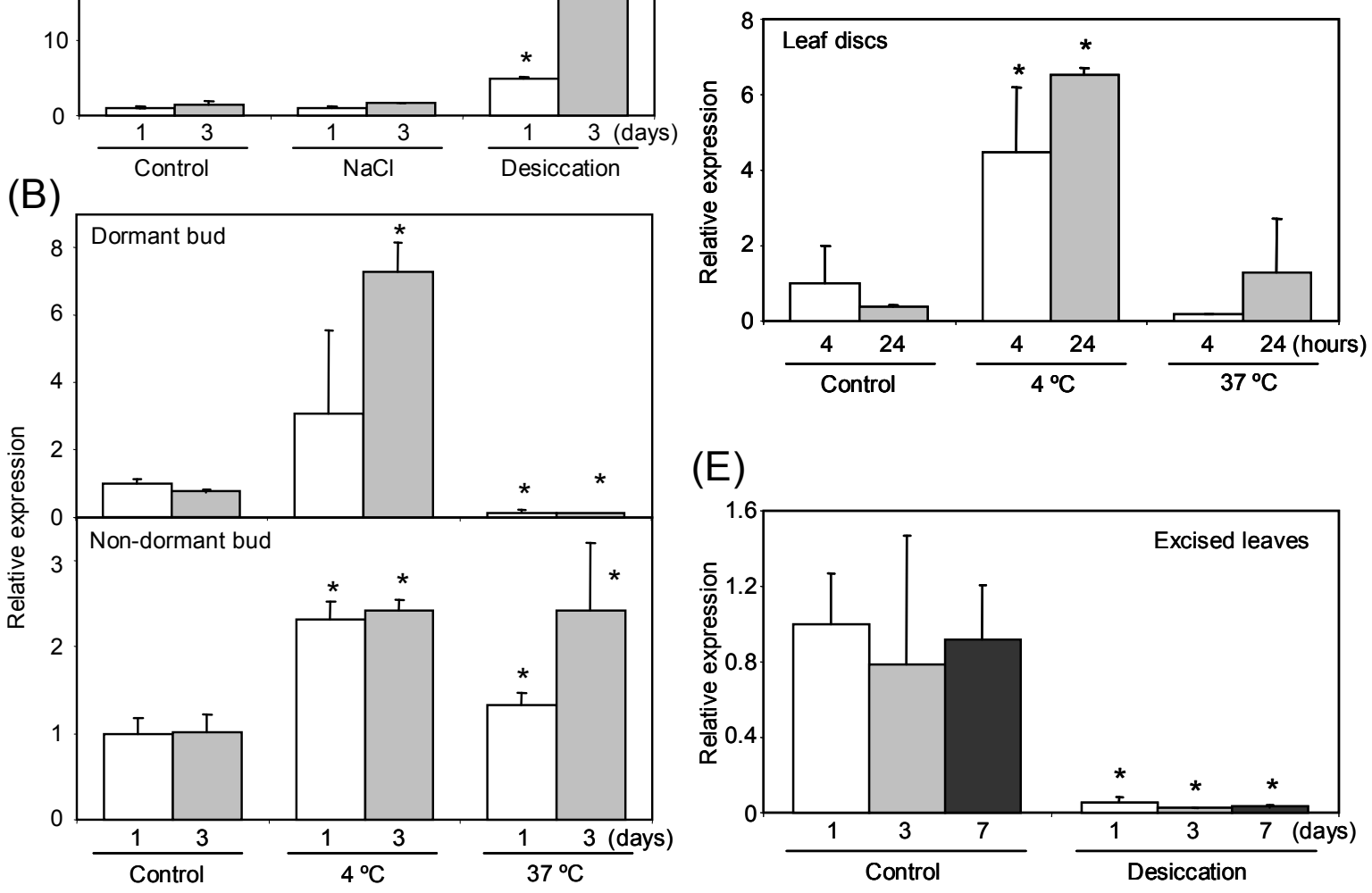

(E)

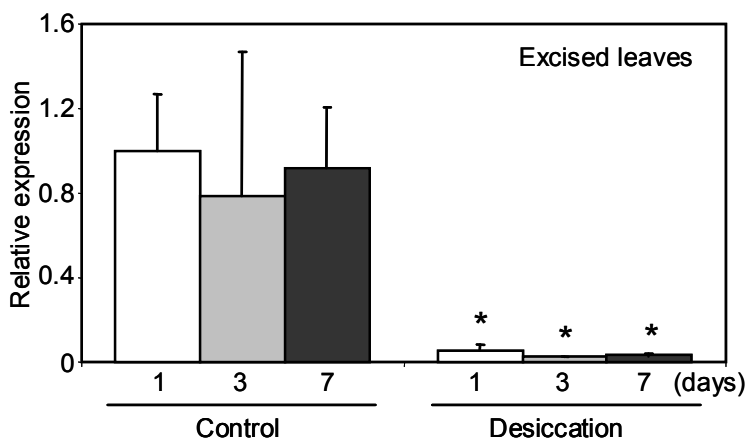

